\title{
Narrowband source of transform-limited photon pairs via four-wave mixing in a cold atomic ensemble
}

\author{
Bharath Srivathsan * * Gurpreet Kaur Gulati:* Chng Mei Yuen Brenda.* \\ Gleb Maslennikov $*$ Dzmitry Matsukevich $\mathbb{\theta}$ and Christian Kurtsiefen
}

\begin{abstract}
We observe narrowband pairs of time-correlated photons of wavelengths $776 \mathrm{~nm}$ and $795 \mathrm{~nm}$ from non-degenerate four-wave mixing in a laser-cooled atomic ensemble of ${ }^{87} \mathrm{Rb}$ using a cascade decay scheme. Coupling the photon pairs into single mode fibers, we observe an instantaneous rate of 7700 pairs per second with silicon avalanche photodetectors, and an optical bandwidth below $30 \mathrm{MHz}$. Detection events exhibit a strong correlation in time $\left(g^{(2)}(\tau=0) \approx 5800\right)$, and a high coupling efficiency indicated by a pair-to-single ratio of $23 \%$. The violation of the Cauchy-Schwarz inequality by a factor of $8.4 \times 10^{6}$ indicates a strong non-classical correlation between the generated fields, while a Hanbury-Brown-Twiss experiment in the individual photons reveals their thermal nature. The comparison between the measured frequency bandwidth and $1 / \mathrm{e}$ decay time of $g^{(2)}$ indicates a transform limited spectrum of the photon pairs. The narrow bandwidth and brightness of our source makes it ideal for interacting with atomic ensembles in quantum communication protocols.
\end{abstract}

PACS numbers: 42.50.-p, 42.65.Hw, 42.50.Ar, 42.65.Lm

Time-correlated photon pairs have been an important resource for a wide range of quantum optics experiments, ranging from fundamental tests [1 [4] to applications in quantum information [5 7]. Most of these applications, however, are based upon manipulation or detection of photons only. More complex quantum information tasks require interfacing of photons to other physical systems, like atoms, molecules, or color centers. A typical example is a quantum network 8], where information is stored or processed in single ions [9], atoms in a cavity 10 13], or in an ensemble of atoms [14 17].

So far, most of the photon pair sources based on spontaneous parametric down conversion in $\chi^{(2)}$ nonlinear optical crystals exhibit a relatively wide optical bandwidth ranging from 0.1 to $2 \mathrm{THz}$ 18, 19]. This makes it difficult to interact with atom-like physical systems, since their optical transitions usually have a lifetime-limited bandwidth on the order of several MHz. Therefore, various filtering techniques have been employed to reduce the bandwidth of parametric fluorescence light. In addition, the parametric conversion bandwidth may be redistributed within the resonance comb of an optical cavity 20 22]. A recent extreme example uses a ring cavity formed by the nonlinear optical medium itself [23].

An alternative approach to this problem is based on four wave mixing (FWM) in an atomic vapor, which resembles the early approaches for entangled photon pair preparation via an atomic cascade decay [2]. In comparison with atomic beam experiments, which had only a very small number of atoms participating in the excitation and decay process at any one time, a cloud of atoms provides a translational symmetry of the nonlinear medium. This leads to momentum conservation or phase matching for the conversion process similar to nonlinear optical interaction in suitable crystalline materials. Momentum conservation in turn allows for a simple collection of the converted light into optical fibers, which leads to a relatively high heralding efficiency of one photon. Correlated photon pairs generated by FWM via cascade decay in a hot ${ }^{85} \mathrm{Rb}$ atomic ensemble have been observed [24, 25], with an optical linewidth of $350 \mathrm{MHz}$ and $450 \mathrm{MHz}$, respectively.

In this paper, we report on spontaneous parametric conversion via FWM in a cold cloud of atoms provided by a Magneto-Optical Trap (MOT), similar to previous work by Channeliere et al. [26]. By doing so the Doppler broadening due to atomic motion is greatly reduced, leading to a bandwidth of the collected fluorescence of the cascade decay that is comparable to the natural atomic line width.

We characterize the temporal properties of the generated photon pairs via a cross-correlation measurement, and the photon statistics of the signal and idler photons from a Hanbury-Brown-Twiss experiment. We also determine the spectral properties of the generated idler photons directly with a scanning Fabry-Perot cavity.

The experimental setup is shown in Figure 1(a). An ensemble of ${ }^{87} \mathrm{Rb}$ atoms is trapped and cooled with a MOT formed by laser beams red detuned by $24 \mathrm{MHz}$ from the $5 \mathrm{~S}_{1 / 2}, F=2 \rightarrow 5 \mathrm{P}_{3 / 2}, F=3$ transition, with a diameter of $\approx 15 \mathrm{~mm}$ and an optical power of $45 \mathrm{~mW}$ per beam. An additional laser tuned to the $5 \mathrm{~S}_{1 / 2}, F=1 \rightarrow 5 \mathrm{P}_{3 / 2}, F=2$ transition optically pumps the atoms back into the $5 \mathrm{~S}_{1 / 2}, F=2$ level. With an axial quadrupole field gradient of $0.3 \mathrm{Tm}^{-1}$ we obtain an atomic cloud with a measured optical density $\left(\mathrm{OD}_{m}\right)$ of $\approx 32$, as determined here and in the following by a fit of the spectral transmission profile of a focused probe beam (waist $125 \mu \mathrm{m}$ ) around the $5 \mathrm{~S}_{1 / 2}, F=2 \rightarrow 5 \mathrm{P}_{3 / 2}, F=3$ transition [27.

To generate the correlated photon pairs, the MOT is turned off, and the atoms are excited to the $5 \mathrm{D}_{3 / 2}, F=3$ level (see Fig. 1(b)) by two orthogonally linearly polarized pump beams (780 $\mathrm{nm}$ and $776 \mathrm{~nm}$ ) intersecting at an 

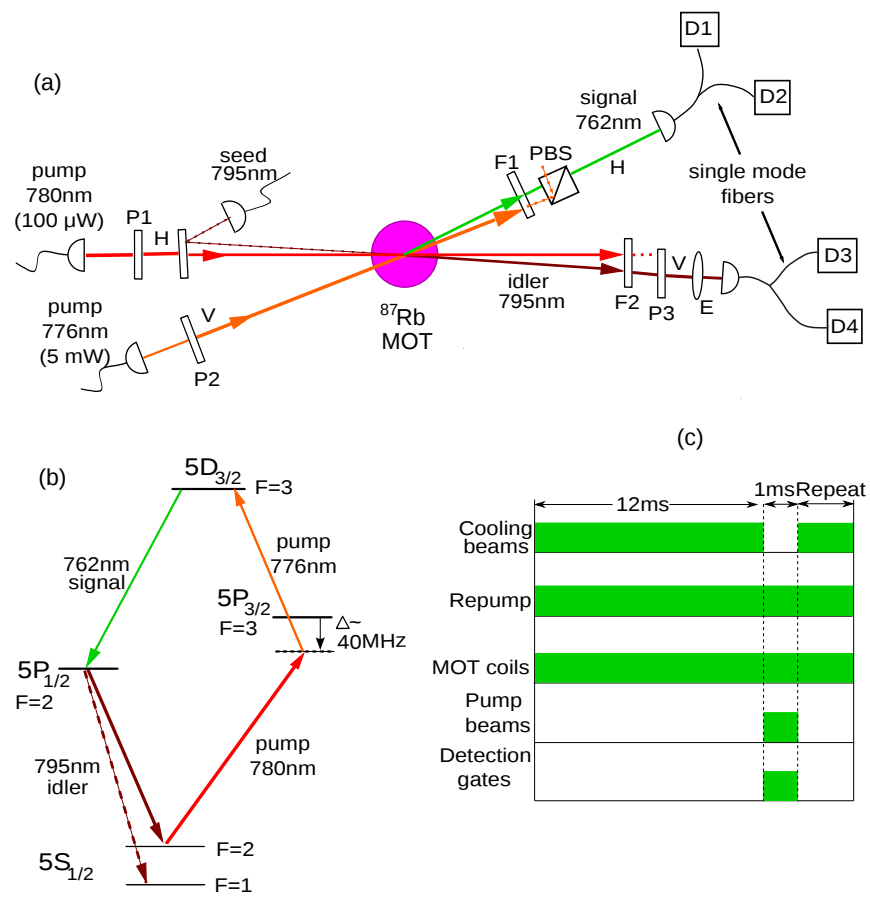

(c)

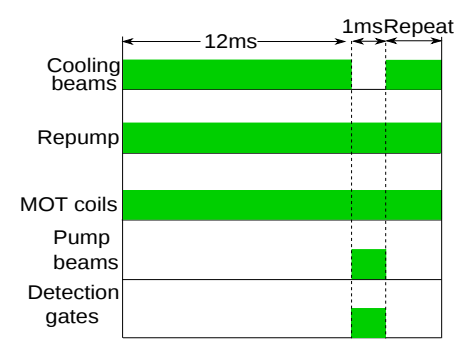

FIG. 1: (a) Schematic of the experimental set up, with P1-P3: polarization filters, F1, F2: interference filters, E: solid etalon, D1-D4: avalanche photodetectors. A $795 \mathrm{~nm}$ seed beam is used to determine the phase-matched direction of coherent $762 \mathrm{~nm}$ emission. (b) Cascade level configuration in ${ }^{87} \mathrm{Rb}$. (c) Timing sequence for one experimental cycle.

angle of $0.5^{\circ}$ in the cold atomic cloud. The $780 \mathrm{~nm}$ pump beam is red detuned by $40 \mathrm{MHz}$ from the intermediate level $5 \mathrm{P}_{3 / 2}, F=3$, since its population would result in a decay back to the initial state.

Experimental periods of $1 \mathrm{~ms}$ for photon pair generation are interleaved with periods of $12 \mathrm{~ms}$ with the MOT turned on to replenish and cool the atomic cloud. This duty cycle was experimentally found to lead to the largest optical density (see Fig. 1(c)).

Photon pairs from a cascade decay of atoms in the excited $5 \mathrm{D}_{3 / 2}, F=3$ level via $5 \mathrm{P}_{1 / 2}, F=2$ back into $5 \mathrm{~S}_{1 / 2}, F=2$ emerge into well-defined directions determined by momentum conservation of the four participant modes. Signal and idler photons generated by parametric conversion are separated from residual pump light by interference filters F1, F2 with a bandwidth of $3 \mathrm{~nm}$ full width at half maximum (FWHM) and a peak transmission of $96 \%$. Uncorrelated photons are further removed from signal and idler modes by a polarizing beam splitter PBS and polarizer P3, where polarizations of pump and target modes are chosen to maximize the product of the Clebsch-Gordan coefficients, and thereby the effective nonlinearity [28]. A temperature-tuned solid fused-silica etalon E (linewidth $375 \mathrm{MHz}$ FWHM, peak transmission $86 \%$ ) in the idler arm is used to remove uncorrelated photons from a decay to the $5 \mathrm{~S}_{1 / 2}, F=1$ level. Paramet- ric fluorescence is then coupled into single mode fibers with aspheric lenses. The effective waists of the collection modes at the location of the cold cloud were determined to be $0.4 \mathrm{~mm}$ and $0.5 \mathrm{~mm}$ for signal and idler by back-propagating light through the fibers and couplers. In an initial alignment step, a seed light at $795 \mathrm{~nm}$ is injected into the idler mode, and coupled into a single mode fiber with an efficiency of $80 \%$. The corresponding signal mode is coupled into the other single mode fiber with an efficiency of $70 \%$.

The photons are detected with Silicon avalanche photodetectors (APDs) D1-D4, (estimated quantum efficiencies of $\approx 40 \%$, dark count rates 40 to $150 \mathrm{~s}^{-1}$ ), and their detection time recorded with a timestamp unit. The combined timing uncertainty of the detectors and timestamping unit is about $0.6 \mathrm{~ns}$.

The histogram of coincidence events $G_{\mathrm{SI}}^{(2)}(\tau)$ as a function of time delay $\tau$ between the detection of signal and idler photons sampled into time bins of width $\Delta \tau=1 \mathrm{~ns}$ is shown in Fig. 2(a). The normalized cross-correlation is defined as

$$
g_{\mathrm{SI}}^{(2)}(\tau)=\frac{G_{\mathrm{SI}}^{(2)}(\tau)}{r_{\mathrm{I}} r_{\mathrm{S}} \Delta \tau T}
$$

where $r_{\mathrm{I}}=2600 \mathrm{~s}^{-1}$ and $r_{\mathrm{S}}=3100 \mathrm{~s}^{-1}$ are the idler and signal photons count rates, and $T$ is the integral time when the pump beams are on, i.e., $1 / 13$ of the total measurement time (Fig. 1(b)). The peak at $g_{\mathrm{SI}}^{(2)}(0)$ of $5800 \pm 76$ indicates a strong temporal correlation. We observe $g_{\mathrm{SI}}^{(2)}(\tau)=1.20 \pm 0.07$ at a time delay of $125 \mathrm{~ns}$ to $1 \mu \mathrm{s}$, with a low decrease to $g_{\mathrm{SI}}^{(2)}(\tau)=1$ at $\tau \approx 100 \mu \mathrm{s}$. The measured $1 / e$ decay time for heralded idler photons from the fit is $6.7 \pm 0.2 \mathrm{~ns}$, which is lower than the single atom spontaneous decay time of $27 \mathrm{~ns}$ from $5 \mathrm{P}_{1 / 2}, F=2$ level. This is due to the superradiance effect in an optically thick atomic ensemble [29, 30].

A total photon pair detection rate $r_{\mathrm{P}}$ of this source can be derived from the measured $G^{(2)}(\tau)$ by integrating over a coincidence time window $\tau_{c}, r_{\mathrm{P}}=\frac{1}{T} \sum_{\tau=0}^{\tau_{c}} G_{\mathrm{SI}}^{(2)}(\tau)$. For $\tau_{c}=30 \mathrm{~ns}$ (vertical lines in Fig. 2(a)), almost all pairs are captured. Under optimal experimental conditions with pump powers of $5 \mathrm{~mW}$ for $776 \mathrm{~nm}, 100 \mu \mathrm{W}$ for $780 \mathrm{~nm}$, and a detuning $\Delta \approx 40 \mathrm{MHz}$ from the intermediate level we obtain $r_{\mathrm{P}}=400 \mathrm{~s}^{-1}$ during the parametric conversion interval. Under these conditions, we find a signal heralding efficiency $\eta_{\mathrm{S}}=r_{\mathrm{P}} / r_{\mathrm{S}}=14.9 \%$, and an idler heralding efficiency $\eta_{\mathrm{I}}=r_{\mathrm{P}} / r_{\mathrm{I}}=23 \%$. By increasing the $776 \mathrm{~nm}$ pump power to $14 \mathrm{~mW}$ and for a detuning $\Delta \approx 20 \mathrm{MHz}$ from the intermediate level, the (instantaneous) pair rate increases to $r_{\mathrm{P}}=7700 \mathrm{~s}^{-1}$, with $g_{\mathrm{SI}}^{(2)}=54 \pm 7$. This corresponds to an average detected pair rate of $592 \mathrm{~s}^{-1}$ including the time during which the pump beams are off. All efficiencies and photon count rates reported are uncorrected for losses due to non-unit detector efficiency, 

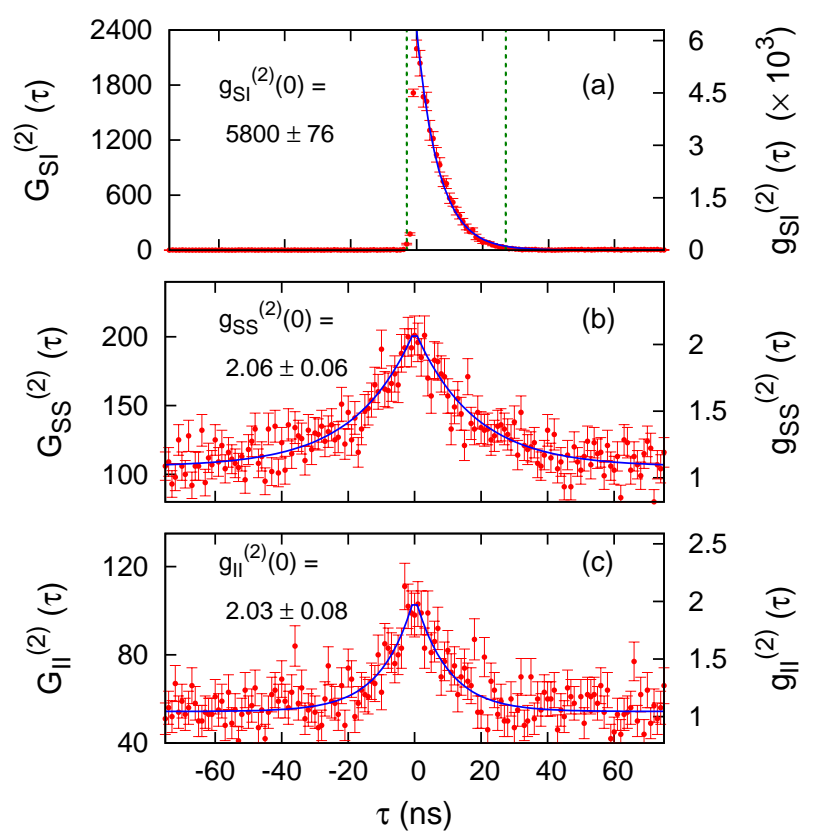

FIG. 2: (a) Histogram of coincidence events $G_{\mathrm{SI}}^{(2)}(\tau)$ for a total integration time of $T=47 \mathrm{~s}$ as a function of time delay $\tau$ between the detection of idler and signal photons sampled into $\Delta \tau=1 \mathrm{~ns}$ wide time bins, and its normalized version $g_{\mathrm{SI}}^{(2)}$ according to Eq. (1). The solid line is a fit to the model $g_{\mathrm{SI}}^{(2)}(\tau)=B+A \times \exp \left(-\tau / \tau_{0}\right)$, where $B=1.20 \pm 0.07$ is the mean $g_{\mathrm{SI}}^{(2)}(\tau)$ for $\tau$ from $125 \mathrm{~ns}$ to $1 \mu \mathrm{s}$, resulting in $A=$ $5800 \pm 76$ and $\tau_{0}=6.7 \pm 0.2 \mathrm{~ns}$. (b) Time resolved coincidence histogram $G_{\mathrm{SS}}^{(2)}(\tau)$ and its normalized version in a HanburyBrown-Twiss experiment on signal photons (detectors D1, D2) for $T=76.3 \mathrm{~s}$. The solid line shows a fit to the model $g_{\mathrm{SS}}^{(2)}(\tau)=C \times\left(1+D \times \exp \left(-|\tau| / \tau_{0}\right)\right)$, resulting in $C=1.08 \pm$ $0.1, D=0.93 \pm 0.06$ and $\tau_{0}=17.8 \pm 1.4 \mathrm{~ns}$. (c) Same as (b) for idler photons detected on D3 and D4 for $T=247.3 \mathrm{~s}$, leading to fit parameters $C=1.04 \pm 0.08, D=0.96 \pm 0.08$, and $\tau_{0}=9.9 \pm 1.2 \mathrm{~ns}$. For all plots, the atomic cloud has an optical density $\mathrm{OD}_{m} \approx 32$.

filtering efficiency and fiber coupling efficiency. Correcting for the detector efficiency on both signal and idler modes, we infer average and instantaneous rates of usable photon pairs coupled into the single mode fibers of about $3700 \mathrm{~s}^{-1}$ and $48000 \mathrm{~s}^{-1}$, respectively.

While it is well-known that light in each of the modes in parametric fluorescence should exhibit thermal photon statistics [31], the coherence time of most photon pair sources is too short to be directly observable in an experiment. Due to the long coherence time of the source presented here, we are able to carry out a direct HanburyBrown-Twiss experiment. The photon counting statistics of signal photons distributed by a fiber beam splitter onto detectors D1 and D2 (Fig. 1(a)) is shown in Fig. 2(b). The normalized $g_{\mathrm{SS}}^{(2)}(\tau=0)=2.06 \pm 0.06$ is compatible with $g^{(2)}(0)=2$ of an ideal single mode ther-

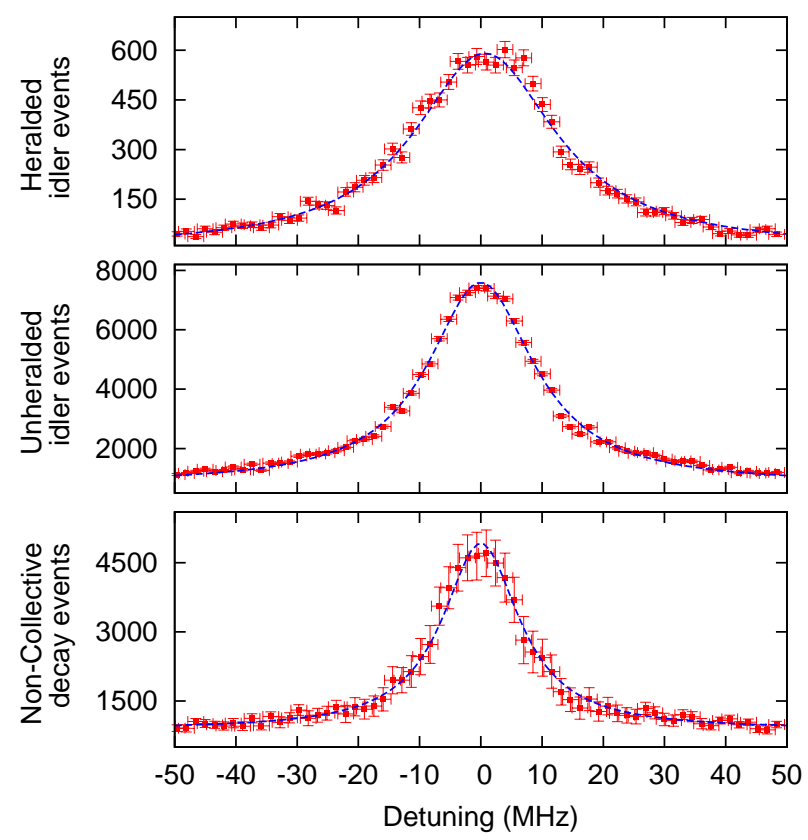

FIG. 3: (a) Spectral profile of idler photons, heralded by the detection of signal photons with an atomic cloud $\mathrm{OD}_{m} \approx 32$. The frequency uncertainty is due to the uncertainty in voltage driving the cavity piezo. The line shows a fit to a model of Lorentzian-shaped photon spectrum, convoluted with the cavity transmission spectrum. The fit gives a bandwidth of $24.7 \pm 1.4 \mathrm{MHz}$ (FWHM). (b) Same as (a), but without heralding. The resulting bandwidth from the fit is $18.3 \pm 1.3 \mathrm{MHz}$ (FWHM). (c) Inferred idler spectrum from a two step (nonsuperradiant) decay with $12.4 \pm 1.4 \mathrm{MHz}$ (FWHM) bandwidth from a fit.

mal state within the statistical uncertainty 32]. From a similar experiment performed on the idler photons, we also observe thermal statistics $\left(g_{\mathrm{II}}^{(2)}(0)=2.03 \pm 0.08\right)$. Without the solid etalon, the idler photons coupled into the single mode fiber are of two different frequencies, thus $g_{\mathrm{II}}^{(2)}(0)<2$ is expected and indeed observed $(1.69 \pm 0.02)$.

The Cauchy-Schwarz inequality bounds the intensity correlation $g^{(2)}$ between two independent classical fields [33, 34]:

$$
R=\frac{\left[g_{I S}^{(2)}(\tau)\right]^{2}}{g_{I I}^{(2)}(0) \cdot g_{S S}^{(2)}(0)} \leq 1
$$

This inequality between the signal and idler fields in our experiment is violated by a factor $R=(8.4 \pm 0.2) \times 10^{6}$ at $\tau=0$ which shows that our source exhibits statistics unexplainable by classical electromagnetic field theory. Our violation factor strongly exceeds the values reported from similar experiments by Du et al. $(R=11600,[35])$ and Willis et al. $(R=495,24)$. We attribute this to lower background counts as compared to what has been observed with gas cells. 
An indirect assessment of the bandwidth of the photons can be obtained from the measured $g^{(2)}(\tau)$, since it is related to the Fourier transform of the spectral distribution. Assuming a transform-limited spectrum, we would infer a bandwidth of $\Delta \nu=1 /\left(2 \pi \tau_{0}\right)=23.8 \pm 0.7 \mathrm{MHz}$ (FWHM) for the heralded idler photons (Fig. 2(a)).

A direct optical bandwidth measurement of idler photons was carried out with a scanning Fabry-Perot cavity (linewidth $2.8 \mathrm{MHz}$ FWHM, measured by a ring-down experiment [36]), tuned $\pm 50 \mathrm{MHz}$ across the $5 \mathrm{P}_{1 / 2}, F=2$ $\rightarrow 5 \mathrm{~S}_{1 / 2}, F=2$ transition. To minimize frequency drift the cavity is temperature stabilized to within $10 \mathrm{mK}$, and kept in vacuum $\left(6 \times 10^{-6} \mathrm{mbar}\right)$. The central transmission frequency is periodically recalibrated via a reference laser locked to the aforementioned atomic transition at $795 \mathrm{~nm}$. The results of this measurement (for $\mathrm{OD}_{m} \approx 32$ of the atomic cloud) is shown in Fig. 3. A fit of the obtained spectrum to a Lorentzian line shape widened by the cavity transfer function leads to a bandwidth of $24.7 \pm 1.4 \mathrm{MHz}$ (FWHM) for the idler photons, if they are heralded by a signal photon (see trace (a)). This is comparable with the bandwidth inferred from the correlation function, indicating that the photons are indeed transform-limited.

However, the observed spectrum of all light in the idler mode (i.e., the unheralded ensemble) shows a narrower bandwidth of $18.3 \pm 1.3 \mathrm{MHz}$ (FWHM). This may be explained by incoherent two step decay (non-collective) contributions to light emitted via the collectively enhanced decay collected in phase-matched directions. The optical bandwidth of light from the collective decay contribution should increase with the atom number $N$ due to an enhanced cascade decay rate, while the bandwidth of light from the two step contribution should remain the same.

The observed bandwidth $\Gamma$ of idler photons (heralded and unheralded) for different atomic densities is shown in Fig. (4) and increases as expected with the $\mathrm{OD}_{m}$, both for the heralded and unheralded photon spectrum.

According to [37, 38], the variation of the emitted bandwidth $\Gamma$ due to collectively enhanced decay can be modeled with the relation $\Gamma=\Gamma_{0}(1+\mu N)$, with the natural line width $\Gamma_{0}=2 \pi \times 5.8 \mathrm{MHz}$ of the $5 \mathrm{P}_{1 / 2}, F=2$ $\rightarrow 5 \mathrm{~S}_{1 / 2}, F=2$ transition [39], the atom number $N$ and a geometry factor $\mu$. We also find a linear increase of $\Gamma$ with $\mathrm{OD}_{m}$ compatible with this model, since $\mu N$ is proportional to our measured $\mathrm{OD}_{m}$; the solid line in Fig. [ shows a fit with the proportionality factor between $\mu N$ and $\mathrm{OD}_{m}$.

Assuming that the incoherent contribution does not significantly contribute to detected pairs at small numerical apertures for collection, we can infer its spectrum by subtracting the heralded idler spectrum from the unheralded idler spectrum after correction for losses in filters $(11 \%)$, optical elements $(7 \%)$, inefficient photodetectors $(60 \%)$, polarization filters $(12 \%)$, and fiber coupling

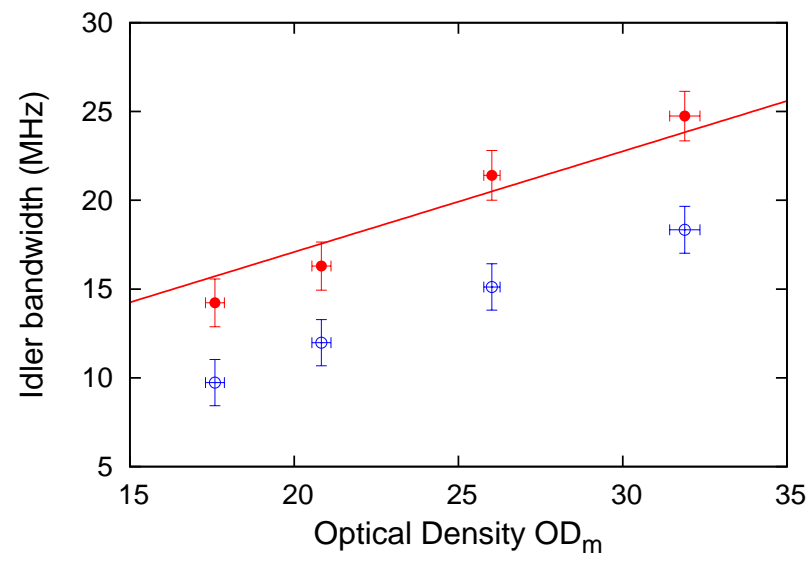

FIG. 4: Bandwidth (FWHM) of heralded idler photons (pairs) for different cloud optical densities $\left(\mathrm{OD}_{m}\right)$ (filled circles). The line shows the theoretical model according to [37, 38]. Open circles indicate the bandwidth (FWHM) of unheralded idler light.

$(30 \%)$. The resulting spectrum for $\mathrm{OD}_{m} \approx 32$ is shown in Fig. 3(c), with a width of $12.4 \pm 1.4 \mathrm{MHz}$ FWHM. This exceeds the natural line width expected for the incoherent two step decay, probably due to self-absorption in the atomic cloud.

In summary, the photon pair source presented in this paper exhibits a high heralding efficiency, is spectrally bright, and shows a narrow optical bandwidth for signal and idler photons. This narrow Fourier-limited bandwidth and the wavelength match with transitions in ${ }^{87} \mathrm{Rb}$, a common workhorse for quantum memories, makes our source a prime candidate for heralded interaction with single atom systems, and quantum memories based on atomic ensembles. The high normalized cross correlation $g_{\mathrm{SI}}^{(2)}$ value clearly indicates the non-classical nature of the photon pairs, and a low background rate. We also demonstrate the thermal statistics of the signal and idler photons from a direct autocorrelation measurement. Beyond correlated photon pair preparation, this scheme can also provide polarization entangled photons by an appropriate choice of pump polarization [24, 26], which can used to implement entanglement swapping and other quantum communication protocols with single atoms [40, 41], ions [42], or atomic ensembles. Furthermore, the long coherence time of our idler photon heralded by the 'click' detection of the signal photon by an APD enables electric field quadrature measurements of the idler photon by homodyne detection using currently available fast photodetectors [43, 44].

We acknowledge the support of this work by the National Research Foundation \& Ministry of Education in Singapore. 
* Center for Quantum Technologies, National University of Singapore, 3 Science Drive 2, Singapore, 117543

$\dagger$ Department of Physics, National University of Singapore, 2 Science Drive 3, Singapore, 117542; Center for Quantum Technologies, National University of Singapore, 3 Science Drive 2, Singapore, 117543

[1] J. F. Clauser, M. A. Horne, A. Shimony, and R. A. Holt, Phys. Rev. Lett. 23, 880 (1969).

[2] A. Aspect, P. Grangier, and G. Roger, Phys. Rev. Lett. 49, 91 (1982).

[3] G. Weihs, T. Jennewein, C. Simon, H. Weinfurter, and A. Zeilinger, Phys. Rev. Lett. 81, 5039 (1998).

[4] E. S. Fry and R. C. Thompson, Phys. Rev. Lett. 37, 465 (1976).

[5] M. A. Nielsen and I. L. Chuang, Quantum Computation and Quantum Information (Cambridge University Press, 2004), 1st ed., ISBN 0521635039.

[6] D. Bouwmeester, A. Ekert, and A. Zeilinger, The Physics of Quantum Information: Quantum Cryptography, Quantum Teleportation, Quantum Computation (Springer, 2010), ISBN 9783642086076.

[7] A. K. Ekert, Phys. Rev. Lett. 67, 661 (1991).

[8] H. J. Kimble, Nature 453, 1023 (2008).

[9] A. Stute, B. Casabone, B. Brandstätter, K. Friebe, T. E. Northup, and R. Blatt, Nature Photonics 7 219(2013).

[10] S. Ritter, C. Noelleke, C. Hahn, A. Reiserer, A. Neuzner, M. Uphoff, M. Muecke, E. Figueroa, J. Bochmann, and G. Rempe, Nature 484, 195 (2012).

[11] T. Wilk, S. C. Webster, A. Kuhn, and G. Rempe, Science 317, 488 (2007).

[12] J. I. Cirac, P. Zoller, H. J. Kimble, and H. Mabuchi, Phys. Rev. Lett. 78, 3221 (1997).

[13] L. Luo, D. Hayes, T. Manning, D. Matsukevich, P. Maunz, S. Olmschenk, J. Sterk, and C. Monroe, Fortschr. Phys. 57, 1133 (2009).

[14] L.-M. Duan, M. D. Lukin, J. I. Cirac, and P. Zoller, Nature 414, 413 (2001).

[15] A. Kuzmich, W. P. Bowen, A. D. Boozer, A. Boca, C. W. Chou, L.-M. Duan, and H. J. Kimble, Nature 423, 731 (2003).

[16] C. H. van der Wal, M. D. Eisaman, A. Andre, R. L. Walsworth, D. F. Phillips, A. S. Zibrov, and M. D. Lukin, Science 301, 196 (2003).

[17] J. K. Thompson, J. Simon, H. Loh, and V. Vuleti, Science 313, 74 (2006).

[18] C. Kurtsiefer, M. Oberparleiter, and H. Weinfurter, Physical Review A 64, 023802 (2001).

[19] F. N. C Wong, J. H. Shapiro and T. Kim, Laser Phys. 16, 1517 (2006).

[20] F. Wolfgramm, X. Xing, A. Cerè, A. Predojević, A. M. Steinberg, and M. W. Mitchell, Opt. Express 16, 18145 (2008).

[21] A. Haase, N. Piro, J. Eschner, and M. W. Mitchell, Opt.
Lett. 34, 55 (2009)

[22] A. Cerè, V. Parigi, M. Abad, F. Wolfgramm, A. Predojević, and M. W. Mitchell, Opt. Lett. 34, 1012 (2009).

[23] M. Förtsch, J. Fürst, C. Wittmann, D. Strekalov, A. Aiello, M. V. Chekhova, C. Silberhorn, G. Leuchs, and C. Marquardt, Nature Communications 4, 1818(2013).

[24] R. T. Willis, F. E. Becerra, L. A. Orozco, and S. L. Rolston, Opt. Express 19, 14632 (2011).

[25] D.-S. Ding, Z.-Y. Zhou, B.-S. Shi, X.-B. Zou, and G.-C. Guo, Opt. Express 20, 11433 (2012).

[26] T. Chanelière, D. N. Matsukevich, S. D. Jenkins, T. A. B. Kennedy, M. S. Chapman, and A. Kuzmich, Phys. Rev. Lett. 96, 093604 (2006).

[27] M. Fox, Quantum Optics: An Introduction, Oxford Master Series in Physics (OUP Oxford, 2006), ISBN 9780198566731.

[28] S. D. Jenkins, D. N. Matsukevich, T. Chanelière, S.-Y. Lan, T. A. B. Kennedy, and A. Kuzmich, J. Opt. Soc. Am. B 24(2), 316 (2007).

[29] R. H. Dicke, Phys. Rev. 93, 99 (1954).

[30] N. E. Rehler and J. H. Eberly, Phys. Rev. A 3, 1735 (1971).

[31] L. Mandel and E. Wolf, Optical Coherence and Quantum Optics (Cambridge University Press, 1995), 1st ed., ISBN 0521417112.

[32] S. Barnett and P. Radmore, Methods in Theoretical Quantum Optics, Oxford science publications (Oxford University Press, USA, 2003), ISBN 9780198563617.

[33] M. D. Reid and D. F. Walls, Phys. Rev. A 34, 1260 (1986).

[34] D. F. Walls and G. J. Milburn, Quantum optics (Springer, 2008).

[35] S. Du, P. Kolchin, C. Belthangady, G. Y. Yin, and S. E. Harris, Phys. Rev. Lett. 100, 183603 (2008).

[36] G. Rempe, R. J. Thompson, H. J. Kimble, and R. Lalezari, Opt. Lett. 17, 363 (1992).

[37] A. Walther, A. Amari, S. Kröll, and A. Kalachev, Phys. Rev. A 80, 012317 (2009).

[38] H. H. Jen, Phys. Rev. A 85, 013835 (2012).

[39] D. Steck, Rubidium 87 D line data (2003), URL http://steck.us/alkalidata/

[40] S. A. Aljunid, B. Chng, M. Paesold, G. Maslennikov, and C. Kurtsiefer., J. Mod. Opt. 58, 299 (2011).

[41] M. K. Tey, Z. Chen, S. A. Aljunid, B. Chng, F. Huber, G. Maslennikov, and C. Kurtsiefer, Nature Physics 4, 924 (2008).

[42] N. Piro, F. Rohde, C. Schuck, M. Almendros, J. Huwer, J. Ghosh, A. Haase, M. Hennrich, F. Dubin, and J. Eschner, Nature Physics 7, 17 (2011), ISSN 1745-2473.

[43] S. Gerber, D. Rotter, L. Slodicka, and J. Eschner, H. J. Carmichael, R. Blatt, Phys. Rev. Lett. 102, 183601 (2009).

[44] A. MacRae, T. Brannan, R. Achal, and A. I. Lvovsky, Phys. Rev. Lett. 109, 033601 (2012). 